\title{
Norois
}

Environnement, aménagement, société

249 | 2018

Patrimoine, vignoble, continuité écologique, transition énergétique, anthropologie des catastrophes

\section{La restauration de la continuité écologique des cours d'eau et la pêche de loisir : héritages, changements et enjeux}

Ecological continuity restoration and the recreational angling: Heritages, changes and challenges

Olivier Thomas et Marie-Anne Germaine

\section{(2) OpenEdition}

Journals

Édition électronique

URL : https://journals.openedition.org/norois/7159

DOI : 10.4000/norois. 7159

ISBN : 978-2-7535-7773-2

ISSN : $1760-8546$

Éditeur

Presses universitaires de Rennes

Édition imprimée

Date de publication : 31 décembre 2018

Pagination : 43-60

ISBN : 978-2-7535-7771-8

ISSN : 0029-182X

Référence électronique

Olivier Thomas et Marie-Anne Germaine, «La restauration de la continuité écologique des cours d'eau

et la pêche de loisir : héritages, changements et enjeux », Norois [En ligne], 249 | 2018, mis en ligne le

31 décembre 2020, consulté le 06 janvier 2022. URL : http://journals.openedition.org/norois/7159 ;

DOl : https://doi.org/10.4000/norois.7159 


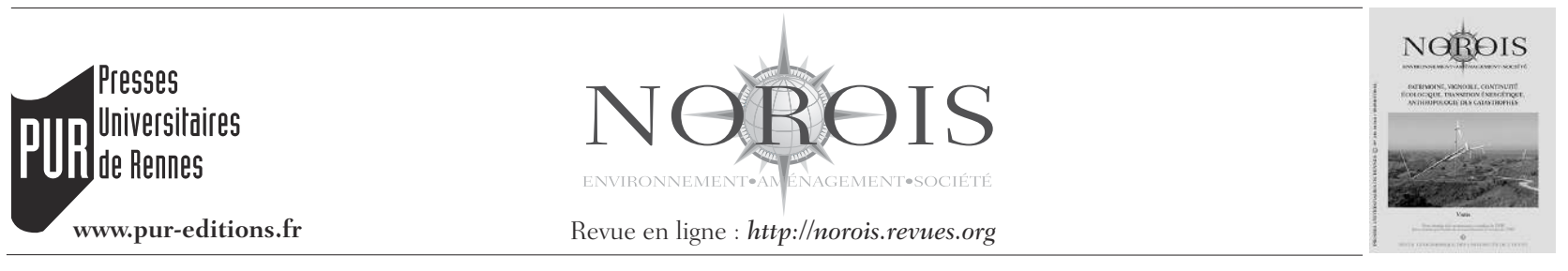

\title{
La restauration de la continuité écologique des cours d'eau et la pêche de loisir : héritages, changements et enjeux
}

\author{
Ecological Continuity Restoration and the Recreational Angling: \\ Heritages, Changes and Challenges
}

\author{
Olivier Thomas a et Marie-Anne Germaine ${ }^{a}$
}

\footnotetext{
a Laboratoire Mosaïques, UMR LAVUE 7218 CNRS - 200 av. de la République, 92000 Nanterre, France.

(olivierthomas.box@gmail.com)(marie-anne.germaine@parisnanterre.fr)
}

\begin{abstract}
Résumé : Dès l'émergence des premières sociétés de pêche à la fin du XIX et au début du Xx ${ }^{e}$ siècle, les pêcheurs à la ligne ont développé une sensibilité à l'égard de l'eau et de la faune piscicole. D’abord mobilisés pour le repeuplement piscicole et la surveillance des cours d'eau, puis pleinement engagés dans la gestion des milieux halieutiques d'eau douce, les pêcheurs vont voir leur rôle évoluer à partir de la Loi-Pêche de 1984. Participant à la protection du patrimoine piscicole et des milieux aquatiques, ils deviennent des acteurs incontournables de la mise en œuvre des nouveaux principes de gestion écologique des cours d'eau, en particulier la restauration de la continuité écologique visant à rétablir la libre circulation des poissons migrateurs. À partir de l'exemple des cours d'eau du Nord-Ouest de la France, et d'un focus sur le cas du démantèlement des barrages de la Sélune, cet article propose de mettre en tension le poids des héritages (pratiques de repeuplement) et les dynamiques de changements (restauration des milieux) qui animent le monde de la pêche de loisir à travers une analyse rétrospective du positionnement des pêcheurs. Il s'agit ainsi d'analyser le rôle ambivalent des acteurs de la pêche, à la fois partenaire et opposant, dans la mise en œuvre de la restauration des cours d'eau. Si les fédérations de pêche prônent le passage vers une gestion patrimoniale, cette posture vient parfois bousculer les habitudes des pêcheurs et plus largement leurs représentations de la nature.
\end{abstract}

Abstract: Since the emergence of the first angling associations in the late $19^{\text {th }}$ and early $20^{\text {th }}$ centuries, anglers have developed a sensibility to water and fish fauna. First mobilized for fish restocking and river watching, then fully engaged in "socio-natural management" (Barthélémy, 2013) of freshwater fisheries environments, anglers will see their role evolve from the 1984 "Loi-Pêche". Participing in the protection of the fish heritage and aquatic environment, anglers are today essential players in the implementation of the new principles of ecological management of rivers and streams, in particular the restoration of ecological continuity aimed at restoring the free movement of migratory fish. Based on the example of rivers in northwestern France, and a focus on the dismantling of the Selune dams, this article proposes to put in tension the weight of traditions (restocking practices) and the dynamics of change (restoration of environments) that animate the world of recreational fishing through a retrospective analysis of fishermen's positioning. In this way, the aim is to analyse the ambivalent role of fisheries stakeholders, both as partners and opponents, in the implementation of river restoration. Although fishing federations advocate a move towards heritage management of stream and fish, this posture sometimes disrupts fishermen's habits and, more broadly, their representations of nature.

Mots clé : DCE, Continuité écologique, pêche, représentation, restauration, savoir

Keywords: EWFD, ecological continuity, fishing, representation, restoration, knowledge 
Au milieu du Xix $x^{\text {e }}$ siècle, le constat du « dépeuplement » des eaux douces en France - bien que non véritablement prouvé (Malange, 2011) - est profondément ancré dans les discours des membres des sociétés savantes (Millet et al., 1856). Sensibilisés à cette situation, les pouvoirs publics s'emparent de la question. La société se souciant alors avant tout de la «part utile de la nature » (Luglia, 2016), l'enjeu est d'abord économique, et c'est de façon très pragmatique "la diminution du revenu des pêches ou la volonté de tirer un profit important des cours d'eau » qui intéresse l'administration (Malange, 2011). Par ailleurs, la gestion des ressources piscicoles est aussi animée par un objectif d'alimentation publique, le poisson étant considéré comme une denrée alimentaire de base pour les populations les plus pauvres (Barthélémy, 2013). Dans ce cadre, diverses mesures, compatibles avec l'industrialisation naissante, vont être prises. Elles concernent la réglementation de la pêche fluviale, l'aménagement des cours d'eau (installation d'échelles à poissons) et la promotion de la pisciculture (Thibault, 1992) qui va dès lors s'inscrire au fondement des pratiques de repeuplement piscicole des cours d'eau en France. Les milieux aquatiques sont alors appréhendés comme « un contenant neutre » (Roux, 2007) qu'il suffirait d'« ensemencer» (Quatrefages, 1862) pour qu'il y ait toujours du poisson à pêcher.

C'est aussi dans la seconde moitié du XIX ${ }^{\mathrm{e}}$ siècle, au cœur de la société industrielle et conjointement à l'essor de la pisciculture, que la pêche à la ligne en tant qu'activité de loisir a émergé en France (Corbin, 2009). En encourageant le développement d'un loisir social, les pouvoirs publics trouvent auprès des pêcheurs le moyen de s'assurer de la réussite des campagnes de repeuplement tout en développant un vaste réseau de surveillance des cours d'eau. Les germes de ce qui deviendra une véritable conscience environnementale (Malange, 2011) au sein du monde de la pêche de loisir sont à rechercher dès cette époque. Très tôt sensibles à la qualité de l'eau, les pêcheurs s'attachent tout au long du $\mathrm{xx}^{\mathrm{e}}$ siècle à dénoncer les pollutions industrielles qui portent atteinte à la faune piscicole. La création du Fishing Club de France (FCF) en 1908, alliance de pêcheurs, de scientifiques et de fonctionnaires derrière le slogan «L'eau pure pour tous! » fonde les mobilisations futures autour de préoccupations écologiques relatives à l'eau, aux rivières, aux poissons et à la pêche. Un véritable militantisme halieutique se développe après la Seconde Guerre mondiale. À partir de 1958, l'Association Nationale de Protection des Eaux et Rivières - Truites Ombres Saumons (ANPER-TOS), composée de pêcheurs et aidée de juristes, va par exemple s'engager à faire réprimer localement les actes de pollution. En défendant les rivières et les poissons, ces pêcheurs militants se font les porte-parole "d'êtres qui ne parlent pas » et agissent pour faire entendre le droit de l'environnement (Gramaglia, 2008). Quelques années plus tard, les pêcheurs contribuent aussi à la loi sur l'eau de 1964 (Bouleau et Barthélemy, 2007; Bouleau, 2009). En 1984, la Loi pêche en fait des acteurs clefs de la protection du patrimoine piscicole et des milieux aquatiques. Ils promeuvent une gestion piscicole patrimoniale : celle-ci consiste à privilégier la réhabilitation du milieu aquatique plutôt qu'à réintroduire des individus via la pratique traditionnelle d'alevinage aujourd'hui contestée (Richard, 1998). Encouragées par l'affirmation d'une gestion intégrée des cours d'eau instaurée par la loi de 1992, les instances nationales de la pêche associative travaillent dès lors à faire évoluer les habitudes de gestion halieutique. Auparavant centrées sur les poissons, ce sont progressivement les cours d'eau - et plus largement les milieux aquatiques - qui se retrouvent au cœur de leurs préoccupations. Cette posture est confortée par la Directive Cadre sur l'Eau (DCE, 2000) puis la Loi sur l'Eau et les Milieux Aquatiques (LEMA, 2006) (Barraqué, 2004; Narcy, 2004 ; Bouleau, 2007; Ghiotti, 2007). Alors que la restauration écologique s'impose en Europe comme en Amérique du Nord comme le nouveau paradigme de gestion des rivières, plusieurs travaux récents ont démontré l'intérêt de s'intéresser aux pêcheurs et à leurs relations aux poissons pour mieux appréhender les enjeux liés à ces nouveaux principes (GottschalkDruscke et al., 2017; Drouineau et al., 2018) ou à la gestion des ressources piscicoles (Martins et al., 2018; Correia Medeiros et al., 2018).

Le long des cours d'eau côtiers du nord-ouest de la France, la mise en œuvre d'opérations de restauration de la continuité écologique constitue pour la pêche associative un outil important. Celle-ci consiste à aménager (passe à poisson ${ }^{1}$ ) voire à sup-

\footnotetext{
1. Une passe à poisson est un dispositif aménagé sur un obstacle naturel ou artificiel (ex : barrage) pour permettre aux poissons - et en particulier aux poissons migrateurs - d'atteindre les zones de reproduction.
} 
primer les obstacles installés en travers des cours d'eau (barrages, seuils et chaussées de moulins) afin de rétablir la libre circulation des poissons notamment migrateurs et d'améliorer la qualité des milieux aquatiques (Adam et al., 2007). Au bord de l'eau cependant, l'effacement d'ouvrages vient parfois bousculer les usages et les représentations de la nature parmi les pêcheurs. À travers l'exemple des régions normande et bretonne, et d'un focus sur le projet d'effacement des barrages de la Sélune (Manche), cet article propose de mettre en tension le poids des héritages et les dynamiques de changements qui animent le monde de la pêche de loisir à l'occasion de la mise en ouvre des politiques de restauration de la continuité écologique. Comment les pêcheurs perçoivent-ils le désaménagement des cours d'eau? Quels rôles jouent-ils dans sa mise en œuvre? Qu'est-ce que cela implique pour eux? Après une présentation des terrains d'étude et de la méthodologie, nous reviendrons sur le processus qui a conduit à l'appropriation de la notion de continuité écologique par le monde de la pêche et nous verrons dans quelle mesure celui-ci participe aux opérations de restauration des cours d'eau. Nous analyserons ensuite les implications de la transformation des milieux halieutiques ${ }^{2}$ pour cerner les fondements des contestations portées par une partie des pêcheurs. Enfin, nous interrogerons l'évolution des représentations de nature parmi les pêcheurs à la ligne afin de mettre en lumière les enjeux qui tendent à émerger pour la pêche associative.

\section{TERrain d'Étude et MÉTHOdologie}

Les régions Normandie et Bretagne sont au cœur de ce travail. Caractérisées par d'importantes façades littorales, elles renferment un dense réseau de cours d'eau côtiers expliquant la présence de nombreux poissons migrateurs (saumon, truite de mer, lamproie, alose...). La majorité des cours d'eau est concernée par des mesures multiples en faveur de leur protection ${ }^{3}$. Ces deux régions dépendent de deux entités hydrographiques, et donc de gestion, distinctes : l'Agence de l'eau Seine-Normandie pour la quasi-totalité de la Normandie et l'Agence

2. L'expression «milieu halieutique » caractérise dans cet article un milieu aquatique perçu et géré en tant que territoire de pêche.

3. Voir à ce sujet le Plan de Gestion des Poissons Migrateurs (PlaGePoMi) Seine-Normandie (2016) et le PlaGePoMi Loire-Bretagne (2013). de l'eau Loire-Bretagne pour la Bretagne. Les enjeux qui pèsent sur la qualité des cours d'eau sont en grande partie liés aux pollutions diffuses d'origine agricole en particulier en Bretagne et dans les zones agricoles les plus intensives de la Normandie. Par ailleurs, et comme l'ensemble des campagnes françaises, les rivières bretonnes et normandes ont été fortement aménagées depuis le Moyen Âge et les ouvrages hydrauliques (vannes, seuils, biefs...) associés aux nombreux moulins installés sur leurs cours constituent aujourd'hui un héritage " encombrant » (Lespez, 2012; Lespez et al., 2017).

La démarche s'appuie sur l'analyse des documents de gestion. En parallèle, entre 2014 et 2017, un travail de terrain a été conduit en Normandie et en Bretagne (figure 1) : il s'appuie sur la réalisation d'entretiens avec des techniciens et des élus des fédérations de pêche $(50,14$ et 61$)$ ainsi qu'avec les directeurs des associations Seine Normandie - Nord Migrateurs (Seinormigr, créée en 2007), Normandie Grands Migrateurs (NGM, créée en 2014) et Bretagne Grands Migrateurs (BGM, créée en 1995) ainsi qu'une approche d'observation participante (réunions d'informations, échanges informels à l'occasion des pêches électriques « Indices d'Abondance Saumon » (IAS) par exemple, participation aux ateliers pêche qui ont accompagné le projet d'effacement des barrages de la Sélune).

La réaction des pêcheurs face aux opérations de restauration de la continuité écologique a été plus particulièrement appréhendée à partir de l'exemple de la Sélune, fleuve côtier qui se jette dans la baie du Mont-Saint-Michel après un parcours de près de $100 \mathrm{~km}$. Cette rivière fait l'objet d'un projet d'envergure inédite puisque le gouvernement a annoncé fin 2009 la suppression des deux barrages hydroélectriques (Roche qui Boit, $16 \mathrm{~m}$ de haut; Vezins, $36 \mathrm{~m}$ de haut) construits au début du $\mathrm{xx}^{\mathrm{e}}$ siècle sur sa partie aval (Germaine et Lespez, 2014). Ce projet visant en particulier la remontée des saumons est l'objet d'une importante controverse dans laquelle la pêche joue un rôle important (Germaine et Lespez, 2017). Le protocole de recherche a fait une large part à l'observation participante. Nous sommes allés à la rencontre des pêcheurs pendant les périodes d'ouverture de la pêche pour observer leurs pratiques, échanger avec eux et saisir leurs relations aux cours d'eau du bassin-versant ainsi qu'à l'estuaire de la Sélune. Des entretiens ont été menés auprès des 


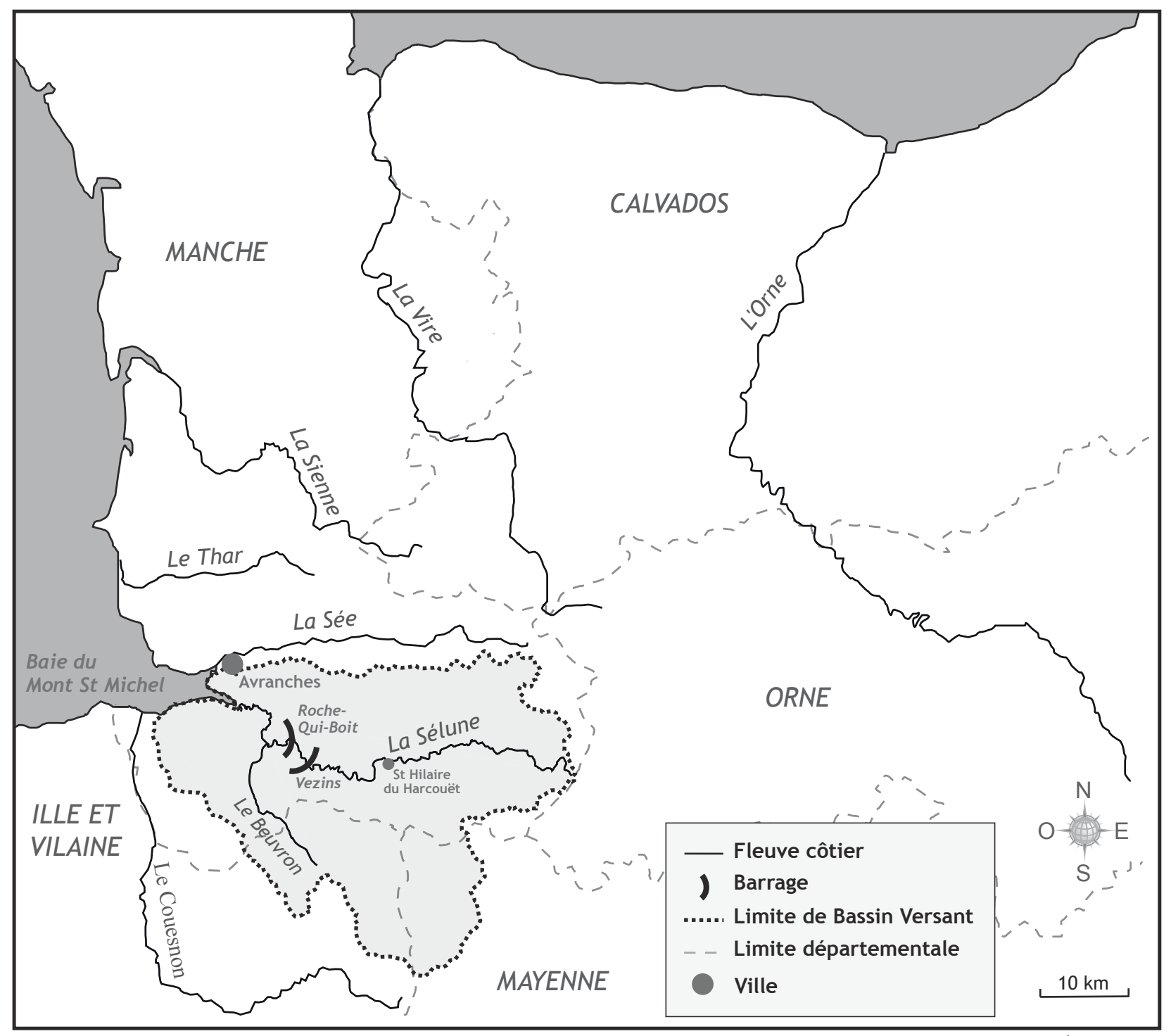

Auteur : Olivier THOMAS, IR, Université Paris Nanterre

Figure 1 : Carte de localisation des cours d'eau bretons et normands étudiés ou mentionnés : le bassin-versant de la Sélune (projet de démantèlement des barrages hydro-éléctriques de Vezins et de la Roche-qui-Boit), l’Orne, la Vire, la Sienne, le Thar, la Sée et le Couesnon Localisation map of rivers and streams studied

présidents des 9 Associations Agréées pour la Pêche et la Protection du Milieu Aquatique (AAPPMA) présentes dans la vallée afin d'appréhender leur implication en matière de gestion piscicole ainsi que les éventuelles problématiques soulevées par des opérations de RCE menées sur les parcours de pêche dont ils ont la gestion.

Par ailleurs, une enquête par questionnaire à destination des pêcheurs français a été menée via Internet entre février et mai 2015. Celle-ci avait notamment pour objectif de connaître l'opinion des pêcheurs au sujet des opérations de restauration de la continuité écologique menées aujourd'hui en France. Parmi les 516 répondants, $35 \%$ habitent dans le département de la Manche et $70 \%$ résident dans les régions Normandie ou Bretagne $^{4}$. Enfin, nous avons collecté les discussions portant sur le devenir des barrages de la Sélune sur différents forums de pêche ${ }^{5}$. Ces deux

4. «Résultats de l'enquête par questionnaire sur la pêche de loisir en eau douce (synthèse) ", Lien : [https://selune.hypotheses.org/718].

5. Stream Dream Forum, Gobages, Club des saumoniers, Pêche Maniac, Fous de pêche, Pêche compétition, Pêche de la truite, Le paradis du carpiste, Forum FFPML, Forum Truite et rivières, Fun flyfishing, Achigan, Carp River Club, Forum Team PCN, jusqu’à fin 2016. 
démarches nous ont permis d'élargir notre étude à des pêcheurs extérieurs à la vallée et d'accéder à des individus supplémentaires que nous n'avons pas pu interroger.

\section{De LA CONTINUité Piscicole À LA CONTINUITÉ ÉCOLOGIQUE}

\section{Concilier la gestion piscicole et le progrès industriel}

Le projet d'aménager les ouvrages transversaux pour permettre la montaison des poissons migrateurs apparait en France au XIX ${ }^{\mathrm{e}}$ siècle. La démarche vise alors à concilier la protection des poissons et par extension les revenus de la pêche fluviale et le progrès industriel. Un premier règlement d'eau est annexé à la circulaire ministérielle du 23 octobre 1851 pour introduire la possibilité d'installer des passes à poissons. Il sera peu appliqué sauf sur quelques cours d'eau fréquentés par les poissons migrateurs. C'est en fait la Loi du 31 mai 1865 qui instaure véritablement l'aménagement d'échelles à poissons sur les barrages hydrauliques dans les textes et fonde l'idée de continuité piscicole. Toutefois, la mise en pratique n'a pas été immédiate, car les décrets d'application ne seront publiés qu'en deux vagues en 1904-1905 (Seine et Loire) et 1921-1924 (Adour, Authie, Canche, Cours d'eau bretons, cours d'eau de Basse-Normandie dont la Sélune) (Thibault, 1992). La construction d'échelles à poissons a préoccupé les scientifiques et le Conseil Supérieur de la Pêche (CSP) tout au long du $\mathrm{XX}^{\mathrm{e}}$ siècle comme en témoignent les nombreuses publications dans le Bulletin Pêche et Pisciculture. Il faut cependant attendre 1984 (Loi Pêche) pour que l'obligation d'efficacité des dispositifs de franchissement piscicole soit posée (art. L.232-6). Les propriétaires doivent alors aménager leur ouvrage dans un délai de 5 ans suivant la publication (en 1986) de l'arrêté « espèces » pour les sections de cours d'eau désignées. Si une partie des pêcheurs, dont la voix est relayée par ANPER-TOS ou l'Association Internationale de Défense du Saumon Atlantique (AIDSA), sont sensibles au fonctionnement des cours d'eau, ce n'est cependant pas l'effacement d'ouvrages qui est visé, mais leur aménagement, et presque toujours uniquement pour les poissons migrateurs. Les migrations d'autres poissons tels que la truite ou le brochet ne sont pas encore au cœur des préoccupations.

Localement, les pêcheurs vont s'emparer très tôt de la possibilité d'installer des dispositifs de franchissement. Ainsi dès 1912, alors qu'un projet de surélévation vient d'être déposé pour réaliser des essais de production hydroélectrique sur la rivière Sélune (Manche, 50), les pêcheurs du Mortainais vont demander que soit installée une échelle à poisson au niveau du seuil du moulin de la Roche-Qui-Boit. Deux ans plus tard, alors que le projet de construction d'un barrage au lieu-dit la Roche-Qui-Boit est engagé, les pêcheurs à la ligne vont à nouveau se mobiliser pour demander l'installation d'une échelle à poissons afin de permettre la montaison des truites et salmonidés migrateurs. Leurs revendications soutenues par le Fishing Club de France $(\mathrm{FCF})^{6}$ ne vont pas aboutir, car le décret d'application de la Loi de 1865 concernant la Sélune ne sera publié qu'en 1924. Quelques années plus tard, lors de l'aménagement de la chute de Vezins en 1927, c’est le ministère des Travaux publics qui va réclamer « pour compenser les difficultés que la présence du barrage apportera aux migrations du poisson et le dépeuplement qui peut en être la conséquence, [que] le concessionnaire fourni[sse] chaque année des alevins dont les espèces et les quantités seront indiquées par ce service, sans que toutefois la dépense correspondant à cette fourniture puisse dépasser la somme de 1000 francs » (art. $7^{7}$ ). Construit en amont du barrage de la Roche-Qui-Boit, le barrage de Vezins mesure 36 mètres de haut. S'il n'est plus question d'aménager un dispositif de franchissement, on observe qu'il s'agit là aussi de concilier gestion piscicole et développement industriel. L'impact des barrages sur les populations de saumons va être abordé de façon quantitative pour mettre en place des mesures compensatoires. Ainsi, en 1978, une étude commandée par le Conseil Supérieur de la Pêche pour tenter de déterminer les dommages causés par les barrages de la Sélune aux populations de saumons atlantiques estime que l'infranchissabilité des ouvrages cause une perte de 20000 smolts $^{8} /$ an :

\footnotetext{
6. Rapport de l'Ingénieur ordinaire des Ponts et Chaussés, Résultats de l'enquête et décisions définitives, 29 avril 1914. Archive DDTM de la Manche.

7. Circulaire No 859 / Décret du 23 novembre 1927 autorisant, déclarant d'utilité publique et concédant les travaux d'aménagement de la chute de Vezins sur la Sélune.

8. Un smolt est un salmonidé juvénile au moment de sa pré-adaptation physiologique et physique à la vie en milieu marin.
} 
la production nécessaire pour y remédier est alors estimée entre 60000 et 80000 smolts d'élevage/an (Porcher, 1978). Par la suite, entre 1989 et 1997, des centaines de milliers de juvéniles de saumons provenant de Bretagne et du sud-ouest de la France vont être déversées dans la Sélune dans le but de contrer le déclin des populations de saumons et de maintenir la pêche de loisir sur ce fleuve côtier (Le Cam et al., 2015). Sur l'Aulne, C. Le Calvez (2015) observe dès la fin du $\mathrm{xx}^{\mathrm{e}}$ siècle des projets similaires (échelles à poissons et repeuplement artificiel) pour favoriser le retour des saumons.

\section{L'émergence de l'idée de "décloisonnement" des cours d'eau dans les années 1990}

En 1984, la Loi-Pêche donne un nouveau sens à la notion de patrimoine piscicole à laquelle est associée l'idée de protection. Le poisson est replacé dans un milieu aquatique qu'il est convenu de préserver. Les associations de pêche doivent quant à elles effectuer des opérations de gestion piscicole. Selon un décret ( ${ }^{\circ}$ 89-804) paru en 1989 et inscrit au Code Rural (Art. L233-3) «l'exercice d'un droit de pêche emporte obligation de gestion des ressources piscicoles. Celle-ci comporte l'établissement d'un plan de gestion ». En 2000, cette obligation est réinscrite au Livre IV « Patrimoine naturel » du Code de l'Environnement (Art. L433-3). Elle incombe aux AAPPMA qui détiennent et usent des droits de pêche. Mais pour assurer une gestion cohérente sur le territoire piscicole associatif et développer un appui technique, ce sont les Fédérations Départementales pour la Pêche et la Protection du Milieu Aquatique (FDPPMA) qui élaborent le document cadre : le Plan Départemental pour la Protection du Milieu aquatique et la Gestion des ressources piscicoles (PDPG) dans lequel figure un diagnostic de l'état des milieux aquatiques et des populations de poissons. Pour rédiger ces documents, les fédérations départementales de pêche recrutent du personnel le plus souvent titulaire d'une licence ou d'un master dans les domaines des sciences de l'environnement. Auparavant dévolues à la gestion des cartes de pêche et à la pisciculture, les fédérations deviennent des pôles techniques et scientifiques au sein du monde de la pêche associative. Une nouvelle lecture des milieux halieutiques se met alors en place dont rend compte la méthodologie des PDPG : «En s’appuyant sur le caractère révélateur des peuplements piscicoles pour la qualité globale des milieux aquatiques, le P.D.P.G. décrit l'état des milieux par "contexte", en diagnostiquant précisément les facteurs limitants » (FDPPMA, 2001). La notion de « contexte» décrit une unité géographique de référence - différente du territoire de pêche des AAPPMA - associée à une espèce cible (ex : la truite) dans laquelle celleci peut réaliser son cycle biologique (reproduction, éclosion, croissance). L'analyse produite à l'échelle du contexte doit révéler ce qui bride ou annihile la fonctionnalité d'un milieu piscicole (bactériologie, piétinement, rejets, barrages, etc.). Dans ce cadre, le « cloisonnement » des milieux causé par la présence d'ouvrages en long (buses) ou transversaux (seuils) est identifié comme un facteur limitant. L'opportunité d'un « décloisonnement » est alors mise en avant comme l'une des mesures à mettre en œuvre pour restaurer les fonctionnalités des habitats aquatiques permettant d'accueillir naturellement les populations de poissons associées. La restauration de la continuité écologique s'impose ainsi comme un des moyens phares de la gestion patrimoniale. Elaborés à la fin des années 1990 et au début des années 2000, les PDPG n'abordent cependant pas encore explicitement, et en ces termes, la question de la restauration de la continuité écologique des cours d'eau.

\section{Le relais assuré par la pêche associative dans la restauration de la continuité écologique}

En 2000, la Directive Cadre Européenne (DCE) impose l'atteinte du bon état écologique des masses d'eau. Aux côtés des critères biologiques et physicochimiques, émergent des critères hydro-morphologiques qui vont motiver la mise en ouvre d'interventions physiques sur les rivières (Germaine et Barraud, 2013; Lespez et al., 2015). Il n'est plus seulement question de lutter contre les pollutions, mais aussi de restaurer les habitats. La suppression des obstacles en travers s'impose rapidement comme un outil phare en Normandie et en Bretagne (Barraud et Germaine, 2017). En 2006, la traduction de la DCE en droit français dans la Loi sur l'Eau et les Milieux Aquatiques (LEMA) rénove la gestion de l'eau, mais aussi l'organisation de la pêche en eau 
douce. Le CSP, qui avait été créé en 1957, disparaît. Il est remplacé par l'Office National de l'Eau et des Milieux Aquatiques (ONEMA, depuis transformé avec la création de l'Agence Française de Biodiversité - AFB). La gestion de la pêche de loisir est quant à elle intégralement confiée au monde associatif. La Fédération Nationale de la Pêche en France et de la Protection des Milieux Aquatiques (FNPF) remplace l'UNPF. Elle se positionne rapidement en faveur de la politique nationale de restauration de la continuité écologique qu'elle juge "essentielle à la reconquête du bon état écologique ${ }^{9}$ ". La LEMA d'abord, puis le Plan d'Action pour la Restauration de la Continuité Écologique (PARCE) en 2009 ouvrent des perspectives aux ambitions de décloisonnement affichés dans les PDPG par les fédérations de pêche. Des financements dédiés peuvent dès lors être engagés par les Agences de l'Eau. À l'échelle départementale, les fédérations de pêche deviennent alors des relais clefs dans la mise en œuvre des opérations d'arasement ou de suppression d'ouvrages.

Localement, au début des années 2000, et dans la perspective de l'élaboration de leurs Plans de Gestion Piscicole (PGP) en cohérence avec le PDPG, des AAPPMA engagent les premiers chantiers de décloisonnement. Mais malgré le soutien de leur fédération, la gestion administrative et le montage budgétaire de tels projets se révèlent souvent difficiles pour les bénévoles associatifs. Aussi, dans le cas du département de la Manche par exemple, la fédération de pêche a choisi de prendre en charge la Maîtrise d'ouvrage des actions de type RCE à la place des AAPPMA. Celles-ci restent cependant impliquées lors des concertations. Elles valident les projets et participent financièrement au budget. Par ailleurs, et pour mener des actions efficaces et cohérentes permettant de retrouver la fonctionnalité piscicole des petits cours d'eau, les projets de décloisonnement se sont construits dans une approche globale comme sur le bassin-versant du Thar où les actions à mener sur les passages busés ou les petits seuils (380 recensés) ont été pensées et traitées dans un même dossier à partir de 2010. D'autres opérations du même type ont été menées sur le bassin du Beuvron ou de la Sienne.

9. Fédération Nationale pour la Pêche en France $>$ Notre rôle et nos missions > Nos prises de position > L'hydroélectricité. Lien : [http://www. federationpeche.fr/148-l-hydroelectricite.htm] (consulté le 10 janvier 2017).
Depuis le PARCE et surtout la révision du classement des cours d'eau (L.214-17) qui impose aux propriétaires d'ouvrages installés sur les cours d'eau classés en liste 2 d'assurer la continuité écologique, les opérations visent des ouvrages plus conséquents (seuils, chaussées voire petits barrages associés à des microcentrales) et la mise en œuvre d'opérations d'arasement est souvent mal perçue (Germaine et Barraud, 2013). Par ailleurs, si la majorité des dossiers d'effacement ou d'aménagement de seuils sont portés par des propriétaires privés, les fédérations départementales de pêche assurent aussi de plus en plus la maîtrise d'ouvrage de certains projets devenant un partenaire clef des agences de l'eau et de l'État dans la mise en œuvre des programmes de restauration. Pour cela, elles sont amenées à devenir propriétaires des sites concernés. Plusieurs projets récents portés par les fédérations de pêche normandes ont ainsi nécessité l'acquisition préalable du foncier. La fédération de pêche du Calvados a ainsi porté en 2016 l'effacement de trois seuils et la démolition de deux anciens sites industriels situés sur l'Orne (sites du Bateau et de la Fouillerie, moulin du Danet) après rachat des sites auprès de leurs propriétaires qui n'en avaient plus l'usage et qui étaient priés de se mettre aux normes. D'un coût total de 2,7 millions d'euros, l'opération a été prise en charge financièrement par l'Agence de l'eau Seine-Normandie (AESN). La fédération de pêche de la Manche a, de son côté, assuré la maîtrise d'ouvrage de plusieurs chantiers de restauration de la continuité écologique sur la Vire par délégation : elle a ainsi pris le relais de l'usine laitière Elvire pour l'arasement du seuil d'Aubigny en juillet 2015 pour un coût de $62000 €$ financé par l'AESN; elle a assuré la maîtrise d'ouvrage du démantèlement du barrage de l'usine de Candol pour l'entreprise Guérin en juillet 2017 avec un financement de $200000 €$ de l'AESN. La fédération de la Manche a en revanche dû acheter la pisciculture de Valjoie sur le Beuvron, affluent de la Sélune, pour assurer en 2015 la suppression du seuil associé. Il arrive également que les pêcheurs soient à l'initiative d'une opération portée par la fédération. Sur la Sienne, le seuil du moulin de Mauny, propriété de l'AAPPMA, a ainsi été supprimé à la demande de l'association locale de pêche. 


\section{RESTAURER LA CONTINUITÉ ÉCOLOGIQUE, OU LA TRANSFORMA- TION DES MILIEUX HALIEUTIQUES}

\section{Une lecture écologique \\ des conséquences de la RCE pour la pêche de loisir}

La présence d'ouvrages en long ou transversaux sur les cours d'eau perturbe le fonctionnement des hydrosystèmes. L'ensemble des conditions environnementales (altitude, pente, vitesse du courant, température, apport en nutriments, débit, largeur du lit, profondeur, turbidité, lumière) qui définissent le continuum amont-aval sont modifiées ce qui introduit des changements au niveau biologique. La rupture de continuité transforme l'organisation du réseau trophique et perturbe l'écosystème de la rivière. La modification de la distribution des différentes espèces de poissons le long de la rivière en est l'une des manifestations. L'effet d'obstacle produit par la présence d'un seuil important ne se limite pas à contraindre la circulation piscicole (et par conséquent l'accès aux zones de reproduction), il modifie aussi les caractéristiques des habitats aquatiques et donc l'étagement des zones piscicoles (Huet, 1949) (figure 2).

Autrement dit, la construction d'un ouvrage (barrage hydroélectrique ou seuil de moulin) a pour conséquence de créer des zones à brèmes ou à barbeaux sur des zones à truites ou des zones à ombres. La présence des barrages de Vezins et la Roche-Qui-Boit sur la Sélune illustre ces perturbations comme en témoigne ce pêcheur et guide de pêche : "La Sélune est une rivière qui est quasiment inversée. C'est-à-dire que la première catégorie est en bas. Moi j'ai assisté à des fraies de carpes à hauteur de Milly [...] sous la distillerie du Coquerel, une fraie de carpe... normal... On est en partie médiane de la rivière [rires] c'est tout à fait normal, c'est leur place... Voilà. Quand on joue à l'apprenti sorcier voilà ce qui peut arriver. » (J., 30 octobre 2014.)

De la même manière, le désaménagement d'un cours d'eau va modifier la physionomie des milieux halieutiques, les peuplements piscicoles et donc l'offre de pêche. Dans le cas des fleuves côtiers, les échanges avec le milieu marin sont restaurés favorisant la recolonisation du cours d'eau par les poissons migrateurs. La liste des espèces susceptibles d'être pêchées s'en trouve ainsi élargie. En recréant le continuum amont-aval, les opérations de restauration de la continuité écologique redistribuent les différentes zones piscicoles sur l'ensemble du linéaire du cours d'eau. La liste des espèces présentes sur les parcours de pêche gérés par les AAPPMA, qui correspondent le plus souvent à des portions de cours d'eau et non au linéaire complet, peut s'en trouver modifiée.

\section{Une transformation de l'offre de pêche diversement appréciée par les pêcheurs}

Si la plupart des pêcheurs à la ligne sont sensibles aux enjeux environnementaux, tout particulièrement en ce qui concerne la qualité de l'eau, la transformation radicale des milieux halieutiques hérités liée à l'effacement d'ouvrages n'est pas toujours bien accueillie. L'abaissement de la hauteur d'eau peut par exemple être mal perçu parce qu'il bouleverse à la fois la distribution des poissons et les habitudes de pêche. En outre, la contestation parmi les pêcheurs semble d'autant plus forte quand le secteur de pêche concerné est accessible et fréquenté depuis longtemps. Ainsi dans le département de la Mayenne, sur l'Ernée, la suppression de petits seuils a été mal accueillie parce qu'elle a induit une perte d'usage importante pour les pêcheurs les moins mobiles ou les familles notamment comme l'explique le président de l'AAPPMA de la Futaie :

"Si là ça avait grogné quand ils avaient enlevé... il y avait des petits barrages qui permettaient toujours de donner... des petits barrages sur l'Ernée [...] qui devaient faire 70 ou $80 \mathrm{~cm}$ qu'ont été enlevés. Là je sais que ça grognait. Bon bah il y a plus d'eau pour pêcher. Il y en avait deux je crois. Deux sur l'Ernée. Moi j'en connais un. On voit encore le... il y a un fossé en béton, puis c'était une plaque avec deux vérins qui faisait une petite retenue. On pouvait pêcher de la carpe, des truites, des gardons... Maintenant, ils ont carrément enlevé le tablier. [...] moi je le fais pour la truite, c'est vrai qu'il y a plus épais d'eau. [...] Mais les gens qui veulent faire de la pêche en famille, ou les anciens qui marchent pas de trop, avec une canne, bah ils peuvent plus parce qu'il n'y a plus où pêcher. Et puis c'était le long des maisons, des jardins. Ces retenues elles servaient 


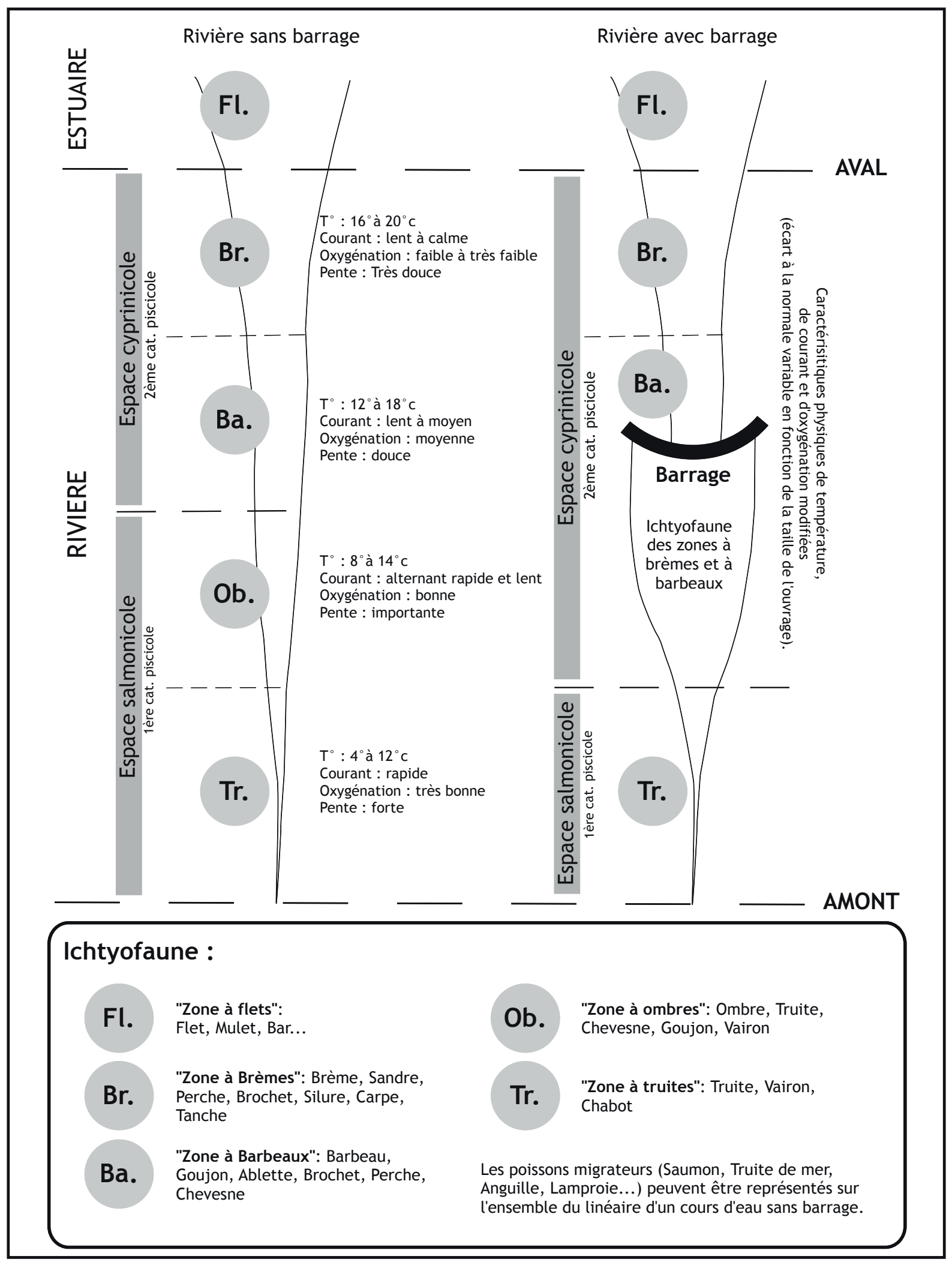

Auteur : Olivier THOMAS, IR, Université Paris Nanterre

Figure 2 : Impact d'un barrage sur l'étagement des zones piscicoles Impact of a dam on fish fauna organisation 
que les gens ils arrosent aussi leurs jardins avec ça. Il $y$ avait plusieurs petits escaliers en parpaings. Je sais que même moi étant gamin j'y allais avec mon grand-père on pêchait les goujons, les vairons... C'est sûr que pour la truite c'est bien, mais après ça élimine toutes les autres espèces. » (24 juillet 2017.)

La modification d'un secteur de pêche et la redistribution de certaines espèces de poissons révèlent aussi l'attachement fort à des lieux perçus comme des espaces hérités et dans lesquels se projette un vécu qui se trouve de fait, suite à la transformation du paysage, relégué au passé :

«[... sur le Couesnon. Ils ont coupé le barrage à Vendel [...] Et il y avait des belles frayères à tanches et tout ça qu'il y avait aussi au moulin de Bleau. C'est pareil là il y avait la vieille rivière qui était là. Sur les barrages de "dans le temps", il y avait une vanne. Ça a toujours existé. Et là, le bief, il allait au moulin. Donc là, il y avait des nénuphars, mais c'était plein de tanches. La tanche elle était dans le Couesnon parce qu'il y en avait beaucoup dans le temps. Il y en a de moins en moins. Elle est en voie de disparition. Là qu'est-ce qu'ils ont fait, bah ils ont coupé le barrage et ça s'en va à l'autre bout. Mais moi je vais vous dire tout ça c'est manvais. C'est tout ce qu'il y a de plus manvais. Parce que moi je pêche le blanc dans le Couesnon. [...] Dans le temps il y avait des nénuphars partout. Fallait voir les gardons qu'on prenait, le blanc tout ça. » (Extrait d'entretien avec un bénévole de La Gaule Fougeraise, 12 août 2016.)

En définitive, les caractéristiques d'un milieu aquatique définissent un territoire et une offre de pêche qui participent à construire sur le temps long une culture de la pêche. Et celle-ci se trouve remise en question, partiellement ou totalement, quand un seuil disparaît. Dans certains cas, comme dans le cadre du projet d'effacement des barrages hydroélectriques de la Sélune, l'incompréhension ou la contestation d'une partie des pêcheurs peuvent muer en véritable opposition.

\section{Le cas du projet d'effacement des barrages de la Sélune}

Annoncé par l'État en novembre 2009 après le Grenelle de l'environnement et à l'occasion du lan- cement du PARCE, le projet d'arasement des barrages de la Sélune trouve ses racines locales dans des délibérations de la Commission Locale de l'Eau, mais il s'agit d'une décision fortement contestée sur le terrain (Germaine et Lespez, 2014, 2017). Dans le monde de la pêche, la perspective d'une transformation radicale des milieux halieutiques dans la vallée est diversement reçue au sein des AAPPMA. En 2011, les promoteurs de l'arasement des barrages de la Sélune fondent le collectif « Les Amis de la Sélune » porté par la fondation Humus (Fonds pour la biodiversité). Ce collectif est composé pour l'essentiel d'acteurs extérieurs au bassin : aux cotés des associations généralistes de protection de l'environnement (France Nature Environnement ou WWF France), on y retrouve des militants halieutiques comme ANPER TOS et des organisations spécialisées dans la défense ou la pêche du saumon (Club des Saumoniers, Association internationale de défense du saumon atlantique, Federation of irish salmon and sea trout anglers, NASF, association bretonne pour la pêche à la mouche). Il s'agit à la fois de faire de la Sélune une des premières rivières à saumons de France et de « retrouver la rivière à saumons d'antan ${ }^{10} »$. L'écotourisme est mis en avant et le saumon doit tout particulièrement attirer pêcheurs et curieux pendant la saison de reproduction. La plupart des communiqués de presse sont rédigés par le directeur de l'Union Régionale des Fédérations de Bretagne-Basse Normandie-Pays de la Loire pour la Pêche et la Protection des Milieux Aquatiques et de nombreuses AAPPMA, voisines, mais extérieures au bassin, sont co-signataires de ces derniers.

En revanche, les présidents des AAPPMA du bassin-versant de la Sélune relatent tous des positions très discutées entre élus du bureau :

« [à propos des barrages] Alors il y a du pour et du contre hein. C'est pas facile d'aborder... là oui j'ai du mal. J'ai du mal même dans mon bureau. Des fois j'ai des gros problèmes avec certains. [...] Ils pêchent les lacs. Faut pas leur enlever leurs poissons. [...] Bah disons ils connaissent bien le lac. Ils connaissent tous les trous, ils connaissent tous les coins. Après va falloir rapprendre ailleurs, c'est

10. Plaquette «Adoptons la vallée de la Sélune » (2011, p. 5) [http://www. selunelibre.org/wp-content/uploads/sites/35/2013/06/PL_SELUNE_V8_ FIN_Decrypted.pdf]. 
moins simple. Bon il y en a qui comprennent aussi. Parce qu'on a des pêcheurs [...] ils sont fervents euh... pour les arasements. » (Extrait d'entretien avec le Président de La Truite Louvignéenne, 12 août 2016)

En conséquence, il est dans la plupart des cas difficile pour les présidents des AAPPMA locales de prendre position pour l'ensemble de leurs membres. Ainsi, sur les 9 associations de la vallée, seule La Truite Saint-Hilairienne, qui souhaite conserver le lac de Vezins (classé en $2^{\text {e }}$ catégorie) situé sur son territoire halieutique, s'est positionnée clairement contre le projet d'effacement des barrages (figure 3).

En amont, la Gaule Mortainaise y est favorable par défaut, son président étant élu au Conseil d'Administration de la Fédération de pêche de la Manche. Ailleurs, les AAPPMA ont fait le choix de la neutralité, à l'image de La Ducéenne à la fois détentrice du droit de pêche sur le lac de la Roche-Qui-Boit (classé en $2^{\mathrm{e}}$ catégorie) et concernée par la pêche du saumon à l'aval des barrages. Initialement favorable au démantèlement, elle a choisi de ne pas se positionner depuis le renouvellement de son bureau en 2015 : «Moi les barrages, ils sont en état, on en profite, c'est très bien. Ils les arasent, on fera avec. " (président, 15 juillet 2016).

Parmi les pêcheurs, la question fait débat. Les différents types de pêches (blancs, carnassiers, carpes) qui se sont développés sur ou au bord des lacs ne pourront pas être pratiqués de la même façon sur la rivière désaménagée. Ce sera même impossible pour les pêches embarquées des carnassiers (bateau ou float tube). En outre, les enquêtes menées auprès des usagers et propriétaires de la centaine de cabanons et pontons de pêche installés le long des lacs témoignent de l'attachement de ces derniers

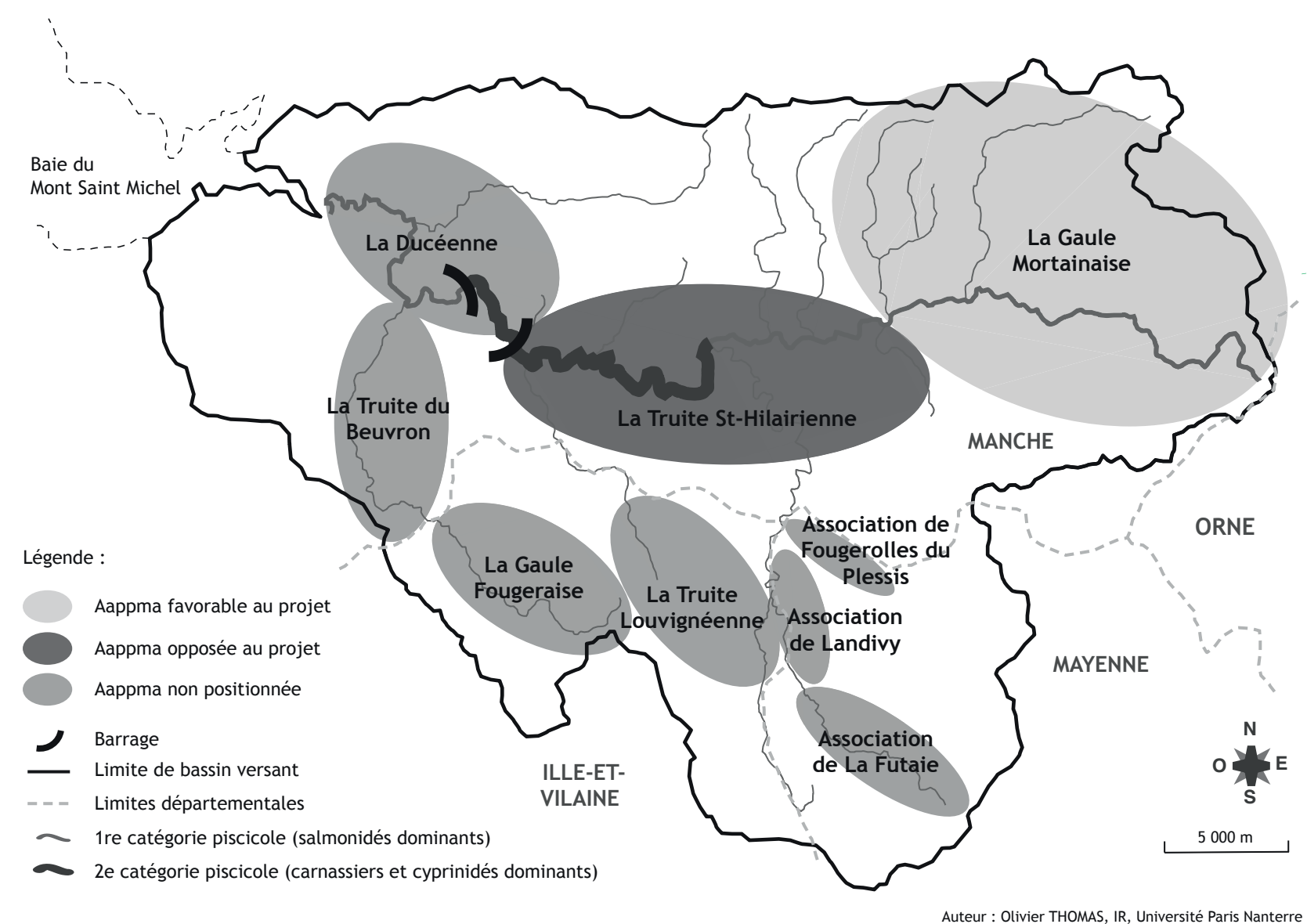

Figure 3 : Les associations de pêcheurs du bassin-versant de la Sélune et le projet d'arasement des barrages Angling associations of the Selune River watershed and the dams removal project 
aux pêches des espèces des milieux cyprinicoles, mais aussi - et tout particulièrement - au paysage des lacs de la Sélune qui constituent pour eux un « décor » irremplaçable (Germaine et al., 2016).

Dans ce contexte, la position de la Fédération de Pêche de la Manche apparait particulièrement inconfortable, puisqu'elle doit composer avec les instances nationales de la pêche de loisir dont la position politique est favorable à l'arasement et tenir compte des voix qui s'élèvent parmi les pêcheurs locaux contre le projet de démantèlement :

"Il faut être honnête. On est, entre guillemets, entre le marteau et l'enclume. [...] On a d'un côté les pêcheurs des lacs et de l'autre côté on est une fédération des associations agréées pour la pêche, mais c'est surtout $90 \%$ de notre travail "protection des milieux aquatiques". Donc avec la nouvelle loi sur l'eau, notre mission a évolué et on a une certaine éthique. On travaille avant tout pour la protection du milieu aquatique. Donc qu'est-ce qui va favoriser la protection des milieux aquatiques : la suppression des barrages. Pour la qualité de l'eau pas seulement pour les poissons. [...] Donc voilà pourquoi on est positionné comme ça. [...] On a une politique au niveau de la fédération, on a une ligne de conduite et on la tient. » (Un technicien, Fédération de pêche de la Manche, 13 avril 2014.)

Sur le terrain, la situation se révèle encore plus complexe puisque la construction de l'opinion au sujet de l'arasement des barrages de la Sélune ne s'inscrit pas dans une logique binaire opposant les « pour » et les « contre » à l'image des résultats de l'enquête par questionnaire menée à une échelle plus large. En effet, 14,3\% des pêcheurs enquêtés disent avoir un «avis partagé » au sujet des opérations de restauration de la continuité écologique menées en France, 8 \% se déclarent "plutôt défavorables » et 10,2\% «plutôt favorables ${ }^{11}$ ». Près d'un pêcheur sur trois aurait ainsi une opinion nuancée (figure 4).

Cette indécision qui est apparue très fréquemment lors du travail d'enquête auprès des pêcheurs de la vallée de la Sélune est particulièrement bien illustrée par la prise de parole d'un pêcheur sur le forum du Carp River Club (CRC) et ce dès 2008. À cette date, l'État n'a pas encore annoncé le démantèlement des barrages, mais le projet a été débattu au sein de la commission locale de l'eau du Schéma d'Aménagement et de Gestion des Eaux (SAGE) du bassin-versant de la Sélune et certains

\section{Au delà du cas de la Sélune, quelle est votre opinion concernant les opérations de restauration de la continuité écologique sur les rivières de France?}

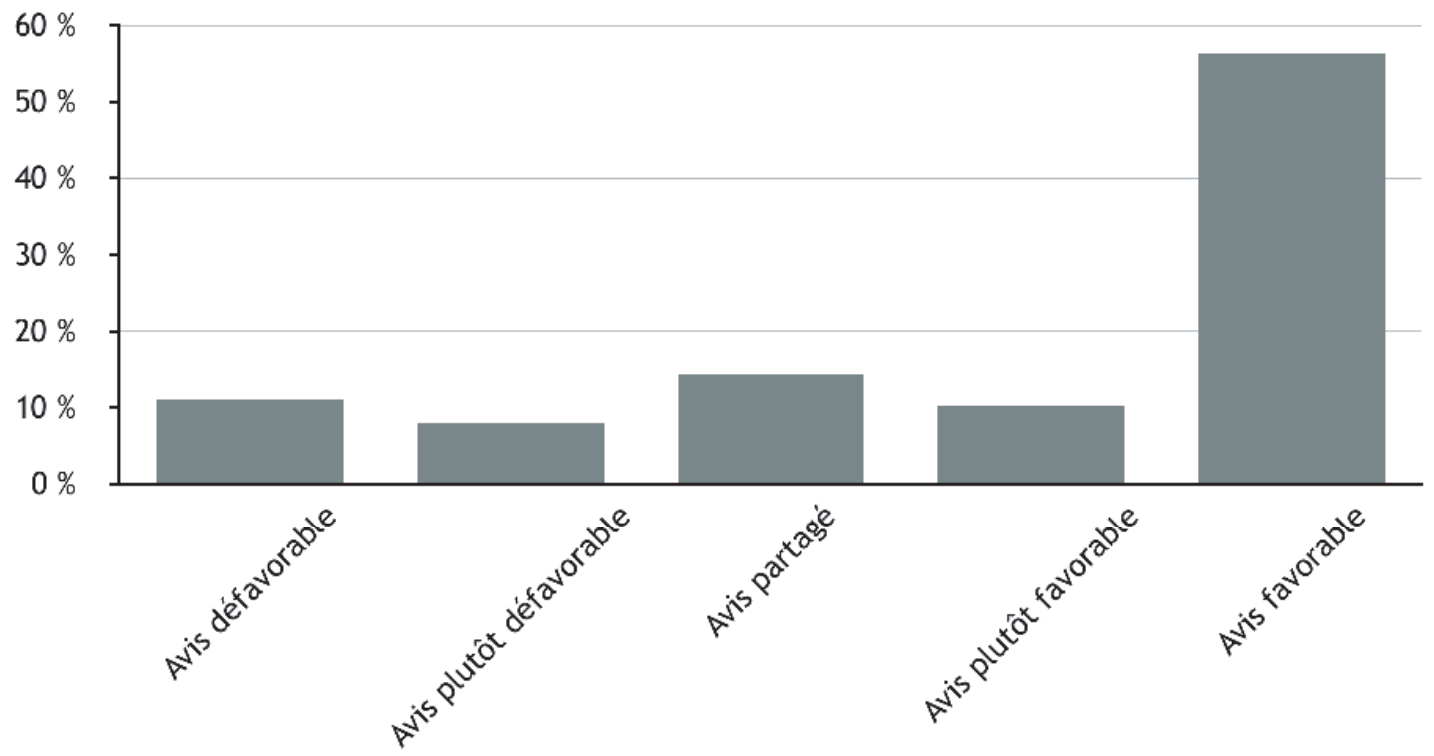

Figure 4 : L'opinion des pêcheurs concernant la restauration de la continuité écologique

Anglers'views about ecological continuity restoration
11. «Résultats de l'enquête par questionnaire sur la pêche de loisir en eau donce (synthèse) », [https://selune.hypotheses.org/718]. 
pêcheurs sensibles aux questions liées à l'eau sont au courant. La diffusion de la nouvelle suscite des réactions dont celle immédiate de se mobiliser pour empêcher la disparition des lacs afin de maintenir l'offre de pêche de la carpe. Beaucoup témoignent de leur attachement au lieu et s'inquiètent déjà du devenir des poissons présents dans les lacs. L'un des membres du forum, informé du dossier et des politiques de gestion qualitative de l'eau, publie un post qui témoigne de la position difficile dans laquelle se trouvent certains pêcheurs des lacs :

«Les solutions pour l'empêcher: AUCUNE! Cette décision est inscrite au SAGE Sélune comme je l'ai déjà dit, ce schéma oriente les actions à effectuer pour atteindre les objectifs de la Directive cadre sur l'Eau (DCE) à l'horizon 2015, la destruction des barrages de Vezins et de la Roche-qui-Boit y est inscrite pour améliorer la qualité de l'eau, rétablir la continuité piscicole et le bon état écologique du milieu. De plus, des financements sont déjà prévus pour ces destructions... alors même avec la plus grande volonté du monde, nous n'y pourrons rien! Cela me fait mal de perdre ce lieu de pêche qui m'a vu grandir, mais je travaille également dans le domaine de l'environnement, qui plus est dans le domaine de l'eau. Il n'existe pas beaucoup de solutions dans ce cas, d'autant plus que la qualité de l'eau sur la Sélune est vraiment manvaise. Pour atteindre les objectifs de la DCE, il faut faire des choix parfois lourds de conséquences et penser à l'avenir et à l'état global de nos cours d'eau plutôt qu'à nos personnes... pour cela il faut bien que certains ouvrages disparaissent: les plus malsains pour l'environnement! Alors même si personnellement et affectueusement cela me touche, je suis avant tout un défenseur de la nature et des écosystèmes et je sais pertinemment que cette masse d'eau (la Sélune) se portera mieux sans les barrages... » (Un membre du Carp River Club, le 18 novembre 2008) ${ }^{12}$.

Du fait de la dimension des barrages, le projet de la Sélune révèle les tensions au sein de la communauté des pêcheurs. La contestation portée par une partie des pêcheurs, de même que l'adhésion d'une autre partie à la restauration de ce fleuve côtier, sont particulièrement saillants et lisibles. Et ce d'autant

12. Forum du Carp River Club (CRC), Dossier « Normandie », Sujet «Barrage de la Roche-Qui-Boit!». [http://www.carperiverclub.com/ forum/index.php?page=post\&ids=6586] (consulté le 20 janvier 2016). plus que la disparition des lacs n'engage pas que des enjeux piscicoles ou halieutiques, mais aussi une transformation des paysages, des usages et plus largement d'un espace de vie pour les pêcheurs qui habitent dans la vallée ou à proximité. Mais ici comme ailleurs, c'est la même problématique qui est mise en lumière : celle d'un bouleversement du rôle des pêcheurs locaux dans la gestion des cours d'eau et plus largement des milieux aquatiques. En effet, et où que soient localisés les projets de restauration de la continuité écologique, la grogne des pêcheurs semble animée par un sentiment d'ingérence écologique sur le territoire halieutique de leurs associations, c'est-à-dire dans des espaces de nature dont ils considèrent être les gestionnaires. Ce sentiment s'exprime à la fois envers les instances de la pêche (fédérations et unions régionales) qui ne leur paraissent pas toujours respecter leur avis ou même s'en préoccuper, mais aussi à l'encontre des collectifs promouvant le retour à des rivières libres ou sauvages impliquant des organisations internationales et s'appuyant sur des porte-parole peu ancrés localement. Ces acteurs ne sont pas considérés comme légitimes à déterminer le devenir de la rivière. Ce sentiment de dépossession est en outre exacerbé par la crainte de projets visant la promotion d'un tourisme halieutique tourné vers le saumon (Salanié et al., 2004) envisagé comme un accaparement de la rivière (et de ses ressources) par des pêcheurs extérieurs.

\section{VERS UNE TRANSFORMATION DES REPRÉSENTATIONS DE NATURE PARMI LES PÊCHEURS?}

\section{Un demi-siècle de localisme associatif}

Que ce soit dans le cadre du développement de l'activité de pisciculture conjointement à celui de la pêche de loisir ou à l'occasion de leurs engagements militants pour la défense du poisson et de la qualité de l'eau, les pêcheurs à la ligne ont très tôt, dès la fin du XIX ${ }^{\mathrm{e}}$ siècle et le début du $\mathrm{Xx}^{\mathrm{e}}$ siècle, développé des liens avec le monde scientifique. Cette posture a contribué à façonner leurs sensibilités aux questions environnementales (Malange, 2011). Mais à partir de 1941, suite à l'instauration de la taxe piscicole, le réseau initialement constitué rassemblant pêcheurs, scientifiques et politiques 
s'est restructuré (Barthélémy, 2013). La création à l'échelle nationale du Comité Central des Pêches puis du Conseil Supérieur de la Pêche (CSP) en 1957 a centralisé les connaissances scientifiques tout en décentralisant l'enjeu de gestion piscicole des rivières et des fleuves sur le territoire. Les fédérations se sont concentrées sur l'activité de pisciculture et la gestion du loisir pêche pendant que les pêcheurs s'enfermaient progressivement dans un « localisme » associatif (Roux, 2007). Le paiement de la redevance pour l'appropriation des lots de pêche a en effet laissé entendre aux pêcheurs qu'ils possédaient leur rivière et qu'ils pouvaient en conséquence y gérer le loisir pêche comme ils l'entendaient. En outre, le fonctionnement associatif et amateur de la pêche de loisir n'imposait pas d'obligation de posséder des compétences particulières en matière d'ichtyologie ou de gestion des milieux aquatiques. En tant qu'Associations Agréées de Pêche et de Pisciculture (AAPP), l'investissement des pêcheurs en matière de gestion piscicole s'est bien souvent résumé aux opérations d'alevinage et de déversement de poissons dits surdensitaires. Mais cette pratique, héritée du projet initial de repeuplement des cours d'eau, s'est progressivement détachée de ses fondements scientifiques. Et à partir de la fin des années 1960 et du début des années 1970, à mesure que le nombre des licenciés en France déclinait, l'activité de gestion piscicole a bien souvent eu moins pour ambition de gérer les populations de poissons que celle des pêcheurs, la vente des cartes de pêche étant au cœur du financement de la pêche associative. En outre, la « logique de propriétaires » (Roux, 2007) qui a caractérisé la gestion du loisir pêche tout au long de la seconde moitié du $\mathrm{Xx}^{\mathrm{e}}$ siècle n'a pas permis aux pêcheurs de s'organiser - ou alors très rarement - à l'échelle des bassins versants, les condamnant à une approche fragmentaire des problèmes environnementaux tout en légitimant la poursuite des opérations d'introduction de poissons nés en pisciculture. En définitive, la période de «sacralisation gestionnaire du pêcheur » (Barthélémy, 2013) a contribué à ancrer des usages et des représentations de nature qui avait émergé au cœur de la société industrielle.

\section{La remise en question des usages hérités}

L'inertie des pratiques de gestion des territoires de pêche en lien avec la pisciculture ou l'incompréhension des pêcheurs vis-à-vis d'opérations de restauration écologique des cours d'eau révèlent le décalage qui s'est opéré entre une partie des pêcheurs et les acteurs engagés dans la gestion de l'eau et de la pêche depuis la fin des années 1990. Le rôle historique des pêcheurs concernant le repeuplement des cours d'eau est par exemple remis en question. La restauration et la protection des milieux aquatiques sont aujourd'hui prioritaires. Cela passe par l'arasement de seuils et la modification des circuits d'alimentation des piscicultures, ce qui bouscule parfois les représentations de la nature parmi les pêcheurs.

«- [Notre pisciculture], on l'a stabilisée. On n'a plus rien tout de suite. On ne sait pas si on va la remettre en route. On a arrêté. »

- Pourquoi vous avez arrêté? Parce que vous avez une pisciculture qui a fonctionné longtemps.

- Qui fonctionne très bien. Mais le problème... toujours pareil... On a une boîte qui s'appelle le SAGE qui veut tout casser. S'ils nous font sauter tous les barrages on n'a plus d'eau nous. On a notre source et on prend l'eau de la rivière. On pompe. Donc s'ils abattent tous les barrages... Parce que tous les petits barrages ils veulent tous les faire sauter. C'est très bien ça... C'est casser la nature. On n'aura plus rien. Nos rivières seront asséchées. " (Extrait d'entretien avec le Président de l'AAPPMA de Saint-Hilaire, 9 août 2016.)

Les actions menées suscitent même parfois l'incompréhension des pêcheurs pour qui seules les opérations d'alevinage permettent de maintenir une population piscicole et donc la pêche sur le territoire dont ils ont la gestion.

"Au Moulin de Mauny, l'Aappma La Sienne gère une écloserie. Donc ils partent de l'œuf et ils déversent des truitelles entre 6 et $8 \mathrm{~cm}$. Et ils ont effectivement la sensation de repeupler le milieu. Et que s'ils faisaient pas ça... alors... [...] Ils ont un taux de conversion de l'œu à la truitelle qui est excellent, de l'ordre de $98 \%$, en partie parce qu'ils utilisent l'eau d'un affluent qui passe devant l'écloserie et qui ne confluait plus la Sienne, parce qu'intégralement capté. [...] Donc projet global RCE, on a retiré le seuil et on a reconnecté cet affluent. On lui 
a refait un lit dans le bief pour qu'il conflue. Cette simple action est probablement plus efficace... bon faudrait quantifier, mais on reconnecte un affluent qui est plutôt pentu, caillouteux et performant comme rivière pépinière... mais ça j'ai beau leur expliquer dans tous les sens, ils l'entendent pas. [...] ça remet en cause ce qu'ils ont toujours fait et puis c'est pas sur le même pas de temps. Ils le voient pas. Un cours d'eau qui se remet à fonctionner, où il y a des frayères, ça peut être long, ça peut ne pas être linéaire, c'est pas si évident que ça. Il peut y avoir des aléas. Et puis les moyens pour prouver quion a une production et la densité... on les a pas! Donc il faut faire un peu confiance. [...] Alors que bah forcément les truitelles dans le bassin ils les voient, ils les comptent... Ils les versent... alors quand ils les versent, celles qui disparaissent en 24 heures ils les reverront pas. Mais ils ont vu ce qu'ils ont mis. Donc comme ils les ont vus, elles existent et celles qu'ils n'ont pas vues, elles n'existent pas. » (Extrait d'un entretien avec un technicien de la fédération de pêche de la Manche, 19 juin 2017.)

À l'occasion de la mise en œuvre des opérations de restauration de la continuité écologique, un dialogue entre le monde scientifique et les pêcheurs à la ligne se remet en place par l'intermédiaire des fédérations départementales de pêche et de protection des milieux aquatiques. Les incompréhensions voire les éventuels conflits que les opérations de RCE font surgir dans le monde de la pêche révèlent le manque de partage des savoirs sur l'environnement et les modalités de gestion de la ressource piscicole. En fait, les objectifs de la restauration de la continuité écologique ne semblent pas plus expliqués aux pêcheurs qu'aux riverains et usagers de la rivière qui se trouvent face au bouleversement de leur environnement quotidien et une certaine remise en cause de leurs pratiques.

\section{L'enjeu de diffusion des savoirs sur les milieux aquatiques}

De la même façon que la pêche de loisir avait pu s'appuyer sur la science à la fin du XIX ${ }^{\mathrm{e}}$ siècle et au début du $\mathrm{xx}^{\mathrm{e}}$ siècle pour multiplier les poissons ou lutter contre les pollutions industrielles, la réalisation de la mission de "protection des milieux aquatiques » qui incombe aujourd'hui au monde de la pêche de loisir s'appuie sur le développement des connaissances dans le domaine des sciences de l'environnement. Mais les logiques de diffusion de ces nouveaux savoirs en direction des pêcheurs sont aujourd'hui plus complexes parce qu'elles ne s'inscrivent plus seulement dans le temps associatif et interpersonnel du loisir pêche, mais aussi, et de plus en plus, dans le cadre de nouveaux espaces d'information sur Internet. Car si la professionnalisation des fédérations départementales de pêche tend à favoriser la sensibilisation des élus au sein des AAPPMA aux enjeux de protection et de restauration des milieux aquatiques, la construction de l'opinion parmi les pêcheurs semble davantage attachée à la maîtrise d'une pluralité de canaux d'informations et en particulier d'Internet. En effet, aux organes officiels de communication d'associations militantes (La Pêche Illustrée pour le FCF ou Truite Ombre Saumon pour l'association nationale de protection des salmonidés) ou de pêcheurs engagés (La Pêche Indépendante, Au bord de l'eau) qui ont fondé la presse halieutique que l'on connait aujourd'hui ( $\mathrm{La}$ pêche et les poissons, Pêches Sportives) se sont ajoutés de nouveaux lieux d'échange et de débat tels que les sites web, les blogs, les forums et les réseaux sociaux. Lors de l'enquête par questionnaire réalisée en 2015, seul un tiers des pêcheurs déclarait encore acheter régulièrement des magazines de pêche, mais ils étaient 59,9\% à dire consulter « souvent » ou « très souvent » des sites ou des blogs de pêche et $54,8 \%$ des pêcheurs disaient participer à un (ou plusieurs) forum(s) de pêche sur internet. Ces espaces d'information et de discussion rassemblent des communautés de pêcheurs autour de techniques (ex : pêche au coup, pêche à la mouche) ou de poissons (ex : carpe, saumon, truite...). Ils constituent aujourd'hui des sources d'acquisition de connaissances sur l'environnement et par extension des médias contribuant à la formation de l'opinion parmi les pêcheurs en matière de gestion piscicole notamment. Par ailleurs, la construction des représentations environnementales et l'appropriation de savoirs sur l'eau et les milieux aquatiques par les pêcheurs amateurs relèvent aussi de socialisations externes au monde de la pêche de loisir. En effet, la pêche n'est pas un loisir « hors société » et le développement d'un discours critique de la part des pêcheurs est aussi à attribuer à la position des individus dans l'espace social hors de la pratique du loisir pêche (Roux, 2007). 
En définitive, et bien que les pêcheurs à la ligne en France aient toujours en commun cette conscience environnementale qui les caractérise et les rassemble, c'est une pluralité de regards et d'opinions qui peut être observée à l'échelle des individus lorsqu'il est question de gestion des milieux halieutiques et plus largement de la pêche. En outre, nous avons pu remarquer qu'en dehors de situations d'entretiens qui constituent des contextes particuliers de formalisation du discours, les pêcheurs rencontrés n'évoquent quasiment jamais la politique de restauration de la continuité écologique actuellement mise en œuvre. Les préoccupations environnementales restent focalisées sur la qualité de l'eau et en particulier les pratiques agricoles jugées souvent seules responsables du dépeuplement des cours d'eau. Ainsi, à l'heure de la mise en œuvre d'actions concrètes visant à réaliser la mission de protection du patrimoine piscicole et des milieux aquatiques qui incombent à la pêche associative, la question de la diffusion et du partage des savoirs sur l'eau et les poissons en direction de tous les pêcheurs apparait comme un enjeu particulièrement important pour redéfinir leur rôle et redonner du sens à leurs actions de gestion piscicole à l'échelle locale notamment.

\section{Conclusion}

La pêche de loisir en France est dans une phase de transition de son histoire. En effet, et alors que le modèle de gestion piscicole hérité de la société industrielle est encore parfois particulièrement présent localement au sein des associations de pêcheurs, un nouveau modèle à vocation patrimoniale a émergé et s'est institutionnalisé. Ce dernier qui s'appuie notamment sur l'amélioration de la qualité de l'eau et la restauration des habitats amène les pêcheurs à repenser leur activité de loisir et plus largement leur rapport aux milieux halieutiques et à la nature. Ainsi, la mise en œuvre d'opérations de restauration de la continuité écologique vient parfois bousculer les usages et les représentations environnementales des pêcheurs à la ligne qui témoignent parfois par la contestation, la critique voire l'opposition radicale de leur attachement aux milieux halieutiques hérités. En outre, ce sont aussi leurs pratiques de gestion de la pêche et des poissons qui sont remises en cause. La transition vers une gestion piscicole patrimoniale oblige en effet à faire autrement en se focalisant moins sur les quantités de poissons que sur la qualité des milieux aquatiques. Le cas de la Sélune étudié dans cet article met en lumière la multiplicité des regards et les positions difficilement conciliables de pêcheurs partagés entre leurs relations individuelles - presque intimes - aux parcours de pêche et les enjeux environnementaux qui accompagnent le projet de restauration de la continuité écologique. Plus largement, et dans chaque territoire halieutique concerné par un projet d'effacement d'ouvrage ou de seuil, c'est une culture de la pêche, étroitement liée à l'histoire de l'AAPPMA, qui se trouve questionnée. En définitive, c'est un enjeu de redéfinition d'une sensibilité écologique partagée parmi les pêcheurs qui semble se dessiner aujourd'hui pour le monde de la pêche. Ce changement qui s'amorce à peine mérite d'être observé parce qu'il donnera probablement aux chercheurs des clés de lecture et de compréhension des usages futurs des cours d'eau et plus largement des vallées en tant qu'espaces vécus par les populations.

\section{Remerciements}

Cet article a été réalisé dans le cadre du programme "Restauration écologique de la rivière Sélune. Paysage, usages, représentations » financé par l'Agence de l'eau Seine-Normandie (AESN) et piloté par M.-A. Germaine (Univ. Paris-Ouest Nanterre-La Défense). Il s'agit d'un des quatre volets du programme de suivi scientifique de l'arasement des barrages de la Sélune piloté par J.-L. Baglinière (INRA). Les auteurs souhaitent remercier l'ensemble des pêcheurs, techniciens et élus qui ont accepté de répondre à leurs sollicitations. Ils tiennent également à remercier les relecteurs anonymes qui, par leurs conseils et remarques, ont permis d'améliorer cet article ainsi que le comité de rédaction de la revue Norois pour son soutien à la publication de ces travaux. 


\section{Bibliographie}

Adam P., Debiais N., Malavoi J.-R., 2007. Manuel de restauration hydromorphologique des cours d'eau, Direction de l'eau, des milieux aquatiques et de l'agriculture (DemaA), Service eaux de surface, Nanterre, Agence de l'eau Seine-Normandie, $60 \mathrm{p}$.

Barraqué B., 2004. Aspects institutionnels de la gestion durable de l'eau en Europe, Cybergeo : European Journal of Geography [En ligne]. DOI : 10.4000/cybergeo.1260.

Barraud R., Germaine M.-A., 2017. Démanteler les barrages pour restaurer les cours d'eau: controverses et représentations, Paris, Quae, coll. «Update Sciences and Technology », $260 \mathrm{p}$.

BarthéLÉmy C., 2013. La pêche amateur au fil du Rhône et de l'histoire. Usages, savoirs et gestions de la nature, L'Harmattan, Paris, $180 \mathrm{p}$.

Bouleau G., 2007. La gestion française des rivières et ses indicateurs à l'épreuve de la directive cadre, Thèse de doctorat, AgroParisTech, 452 p.

Bouleau G., 2009. La contribution des pêcheurs à la loi sur l'eau de 1964, Économie Rurale, n³09, p. 9-21. DOI : 10.4000/economierurale.261.

Bouleau G., Barthélémy C., 2007. Les demandes sociales de restauration des rivières et leurs traductions scientifiques et politiques, Techniques-Sciences-Méthodes (TSM), n $\mathrm{n}^{\circ}$, p. 66-76.

Corbin A., 2009. L'avènement des loisirs 1850-1960, Ed. Flammarion, Paris, 626 p.

Correia Medeiros M., Duarte Barboza R.-R., Martel G., Da Silva Mourao J., 2018. Combining local fishers' and scientific ecological knowledge, Implications for comanagement, Ocean \& Coastal Management, vol. 158, p. 1-10.

Drouineau H, Carter C., Rambonilaza M., Beaufaron G., Bouleau G., Gassiat A., Lambert P., Le Floch S., Tétard S., de Oliveira E., 2018. River continuity restoration and diadromous fishes: much more than an ecological issue, Environmental Management. DOI: 10.1007/s00267017-0992-3.

FDPPMA (Fédération de la Manche pour la Pêche et la Protection du Milieu Aquatique), 2001. Plan Départemental pour la Protection du Milieu Aquatique et la Gestion des ressources piscicoles de la Manche, AESN, CG de la Manche, CSP, DDAF 50, CATER, Parc Naturel Régional des Marais du Cotentin et du Bessin, 189 p. (non publié)

Germaine M.-A., Barraud R., 2013. Les rivières de l'Ouest de la France sont-elles seulement des infrastructures naturelles? Les modèles de gestion à l'épreuve de la DCE, Natures, Sciences, Sociétés, no 4, vol. 21, p. 373-384. DOI : $10.1051 / \mathrm{nss} / 2014003$.

Germaine M.-A., Lespez L., 2014. Le démantèlement des barrages de la Sélune (Manche). Des réseaux d'acteurs au projet de territoire?, Développement durable et territoires [En ligne], no 3, vol. 5. DOI : 10.4000/developpementdurable. 10525 .
Germaine M.-A., Viry M., Menozzi M.-J., 2016, Construction des lieux et rapports à la nature, Norois [En ligne], nº 240. DOI : 10.4000/norois.5955.

Germaine M.-A., Lespez L., 2017. The failure of the largest project to dismantle hydroelectric dams in Europe? (Sélune River, France, 2009-2017), Water Alternatives, n 3, vol. 10, p. 655-676.

Gнiotтi S., 2007. Les territoires de l'eau: gestion et développement en France, CNRS éditions, Paris, 246 p.

Gottschalk-Druscke C., Lundberg E., Drapier L., Hychka K.C., 2017. Centering fish agency in coastal dam removal and river restoration, Water Alternatives, $\mathrm{n}^{\circ}$ 10, vol. 3, p. 724-743.

Gramaglia C., 2008. Des poissons aux masses d'eau : les usages militants du droit pour faire entendre la parole d'êtres qui ne parlent pas, Politix, no 83, vol. 2008/3, p. 133-153. DOI : 10.3917/pox.083.0133.

Huet M., 1949. Aperçu des relations entre la pente et les populations piscicoles des eaux courantes, Revue Suisse d'Hydrologie, vol. 11, no 3/4, p. 332-351.

Le Cam S., Perrier C., Besnard A.-L., Bernatchez L., Evanno G., 2015. Genetic and phenotypic changes in an Atlantic salmon population supplemented with non-local individuals: a longitudinal study over 21 years, Proceedings of the Royal Society B, $\mathrm{n}^{\circ}$ 1802, vol. 282. DOI: 10.1098/ rspb.2014.2765.

Le Calvez C., 2015. Rétablir la libre circulation piscicole dans les vallées fluviales : mise en perspective des enjeux et des aménagements à partir du cas de l'Aulne (XIX ${ }^{\mathrm{e}}-\mathrm{XXI}^{\mathrm{e}}$ siècles), Norois, $\mathrm{n}^{\circ} 237$, p. 33-50.

Lespez L., 2012. Paysages et gestion de l'eau. Sept millénaires d'histoire de vallées et de plaines littorales en Basse-Normandie, Bibliothèque du pôle rural no 3 , Presses Universitaires de Caen/MRSH, 336 p.

Lespez L, Beauchamp A., Germaine M.-A., Barraud R., 2017. De l'aménagement au désaménagement : les temps de l'environnement des systèmes fluviaux ordinaires de l'ouest de la France, in Barraud R., Germaine M.-A. (dir.), Démanteler les barrages pour restaurer les cours d'eau, Controverses et représentations, Paris, Quae, coll. «Update Sciences and Technology », p. 27-42.

Lespez L., Viel V., Rollet A.-J., Delahaye D., 2015. The anthropogenic nature of present-day low energy rivers in western France and implications for current restoration projects, Geomorphology, vol. 251, p. 64-76.

Luglia R., 2016. Gérer et protéger la nature. Les relations entre naturalistes, pouvoirs publics et agriculture. France $\mathrm{XIX}^{\mathrm{e}}-\mathrm{XXI}^{\mathrm{e}}$ siècle. MRSH, Pôle Société et espaces ruraux, Conférence enregistrée, La Forge Numérique. [http://www.unicaen.fr/ recherche/mrsh/forge/3915].

Malange J.-F., 2011. Histoire sociale des pratiques de pêche à la ligne en France de 1829 à 1941 : aux origines d'une conscience environnementale, Thèse de doctorat en Histoire, Université de Toulouse 2 Le Mirail, 753 p.

Martins I.M., Pereira Medeiros R., Di Domencio M., HanAzAKI N., 2018. What fishers' local ecological knowledge can reveal about the changes in exploited fish catches, Fisheries Research, 198, p. 109-116. 
Millet C., de Montgaudry M., Perier A., Wallut C., 1856. Rapport sur les mesures à prendre pour assurer le repeuplement des cours d'eau de la France, Bulletin de la Société Impériale Zoologique d'Acclimatation, p. 223-224.

Narcy J.-B., 2004. Pour une gestion spatiale de l'eau. Comment sortir du tuyau?, Bruxelles, PIE/Peter Lang, coll. « Ecoplis », $\mathrm{n}^{\circ} 4,342 \mathrm{p}$.

Porcher J.-P., 1978, Essai de détermination des dommages causés par les barrages de la Roche-qui-Boit et de Vezins aux populations de Saumon Atlantique (Salmo Salar L.), Conseil Supérieur de la Pêche, $7 \mathrm{p}$.

Quatrefages (DE) J.L. A., 1862. Fertilité et culture de l'eau, Paris, Imprimerie de L. Martinet, 32 p.

Richard A., 1998, Gestion piscicole : Interventions sur les populations de poissons, repeuplement des cours d'eau salmonicoles, Mise au point, Conseil Supérieur de la Pêche, 256 p.
Roux F., 2007. Des « pêcheurs sans paniers ». Contribution à une sociologie des nowveaux usages culturels de la nature, Thèse de sociologie, Université de Nantes, 358 p.

Salanié J., Le Goffe P., Surry Y., 2004. Évaluation des bénéfices procurés par le démantèlement des barrages hydroélectriques : le cas de la pêche au saumon sur la Sélune, Ingénieries, $\mathrm{n}^{\circ} 39$, p. 65-78.

Thibault M., 1992. Analyse historique de la mise en place des mesures de gestion des ressources halieutiques des eaux continentales, Rencontres halieutiques de Rennes, Les eaux continentales françaises : une richesse à gérer, ENSA Rennes, p. 15-33. 